\section{Dr. Petri, et al reply}

To the Editor:

We thank The Journal for this opportunity to restate some of the findings in our report ${ }^{1}$.

One of our conclusions is that antiphosphatidylserine/prothrombin $(\mathrm{aPS} / \mathrm{PT})$ is associated with thrombosis in systemic lupus erythematosus (SLE), agreeing with the data published by Bertolaccini, et $a l^{2}$. We completely agree that aPS/PT should be considered as an additional test for antiphospholipid syndrome (APS) and should not be considered just a replacement for lupus anticoagulant (LAC). While most, but not all, aPS/PT-positive specimens are LAC-positive, not all LAC-positive specimens are aPS/PT-positive. Although the validity of LAC testing in anticoagulated patients might be debatable, our suggestion that aPS/PT IgG/IgM tests "might serve as a useful alternative to lupus anticoagulant" in these situations is reasonable.

We did not focus on comparing LAC-positive versus LAC-negative patients. Our table data ${ }^{1}$ compared thrombosis versus no thrombosis, stroke versus no stroke, etc. Our tables were not univariate analyses, but as stated in the footnote for each table, were adjusted for age, sex, and ethnicity. Additional analysis, adjusting for hypertension and hyperlipidemia, did not result in different conclusions.

Our focus was on 2 "novel" assays, aPS/PT and IgA antiphospholipid assays. Our work on anti- $\beta_{2}$-GPI and thrombosis has been published previously $^{3}$, and therefore was not the focus of the report.

The assays (aPS/PT and IgA) are not affected by anticoagulation. The Russell's viper venom time test (RVVT) is, in our center, prolonged in anticoagulated patients. The RVVT confirmatory test may be valid (but can still be falsely positive in those with supratherapeutic international normalized ratios). We believe that alternatives to clotting assays are needed in the evaluation for antiphospholipid status.
MICHELLE PETRI, MD, MPH, Professor of Medicine, Director, Johns Hopkins Lupus Center, Baltimore, Maryland; GARY L. NORMAN, PhD; WALTER BINDER, PhD; ZAKERA SHUMS, MS, Inova Diagnostics Inc., San Diego, California, USA; EHTISHAM AKHTER, MD, MPH; HONG FANG, MD, MS, Johns Hopkins University. Address correspondence to Dr. M. Petri, Johns Hopkins University, Rheumatology, 1830 E. Monument Street, Suite 7500, Baltimore, MD 21205, USA.

E-mail: mpetri@jhmi.edu

\section{REFERENCES}

1. Akhter E, Shums Z, Norman GL, Binder W, Fang H, Petri M. Utility of antiphosphatidylserine/prothrombin and IgA antiphospholipid assays in systemic lupus erythematosus. J Rheumatol 2013;40:282-6.

2. Bertolaccini ML, Sciascia S, Murru V, Garcia-Fernandez C, Sanna G, Khamashta MA. Prevalence of antibodies to prothrombin in solid phase (aPT) and to phosphatidylserine-prothrombin complex (aPS/PT) in patients with and without lupus anticoagulant. Thromb Haemost 2013;109:207-13.

3. Mehrani T, Petri M. Association of IgA Anti-beta 2 glycoprotein I with clinical and laboratory manifestations of systemic lupus erythematosus. J Rheumatol 2011;38:64-8.

J Rheumatol 2013;40:9; doi:10.3899/jrheum.130468 